\title{
Anti-leucine-rich glioma-inactivated 1 limbic encephalitis: A case report and literature review
}

\author{
JINGYAO LIU ${ }^{1}$, MIN LI $^{1}$, GUIBO LI ${ }^{2}$, CHUNKUI ZHOU ${ }^{1}$ and RENSHENG ZHANG ${ }^{1}$ \\ ${ }^{1}$ Department of Neurology, First Hospital, Jilin University; ${ }^{2}$ Changchun Medical College, Changchun, Jilin 130031, P.R. China
}

Received October 12, 2014; Accepted September 1, 2015

DOI: $10.3892 /$ etm.2015.2866

\begin{abstract}
This study describes the case of a 41-year-old woman admitted for anterograde memory loss, right facial grimacing and right arm posturing that had begun 1 month previously. Cranial magnetic resonance-diffusion weighted imaging and -fluid-attenuated inversion recovery imaging revealed a hyperintense signal in the left hippocampus and right basal ganglia, but no contrast enhancement. An electroencephalogram revealed rhythmic sharp and slow waves and rhythmic $\theta$ build-ups in the left temporal area. Single-photon emission computed tomography showed increased regional blood flow perfusion in the left cerebral frontal lobe and the right basal ganglia. The cerebrospinal fluid was normal, with the exception of the presence of leucine-rich glioma-inactivated 1 (LGI1) antibodies, and LGI1 antibodies were also found in the blood serum. The presence of the antibodies, the faciobrachial dystonic seizures (FBDSs) and the memory loss indicated limbic encephalitis. After 3 months of immunotherapy, the patient was free from epileptic seizures and had undergone a partial memory restoration. FBDSs alone justify the immediate initiation of immunotherapy, even prior to laboratory confirmation of the disease, as early treatment limits the duration of the illness.
\end{abstract}

\section{Introduction}

Anti-leucine-rich glioma-inactivated 1 (LGI1) limbic encephalitis (LE) is characterized by faciobrachial dystonic seizures (FBDSs), memory loss and antibodies against the leucine-rich, glioma-inactivated subunit of the voltage-gated potassium channel (VGKC)-complex. FBDSs precede the other symptoms and prompt the diagnosis (1). The clinical course is subacute (2). Newly discovered antibodies have revealed that target neuronal cell surface antigens, VGKCs and ligand-gated

Correspondence to: Professor Rensheng Zhang, Department of Neurology, First Hospital, Jilin University, 3302 Jilin Street, Changchun, Jilin 130031, P.R. China

E-mail: 415413119@qq.com

Key words: leucine-rich glioma-inactivated 1, limbic encephalitis, faciobrachial dystonic seizures ion channels are present in anti-LGI1 LE, despite not being commonly found in the presence of malignancies (3). LGI1 has also been identified as a specific target of potassium channel antibodies (4). In the present case, the patient presented with FBDSs and memory loss, but no nausea, vomiting, insomnia or hyponatremia, which commonly accompany anti-LGI1 LE.

The patient provided informed consent for the use of her case data, and permission to present the case was obtained from the ethics committee of the First Hospital of Jilin University (Changchun, China).

\section{Case report}

A 41-year-old woman was admitted for anterograde memory loss and right facial grimacing and arm posturing that had started 1 month previously, in May 2014. The relatives of the patient stated that her memory disturbances, particularly short-term memory loss and disorientation, had developed gradually over the last month. Episodes of involuntary movement of her right arm and face, lasting between 10 and $30 \mathrm{sec}$, were occurring with increasing frequency; while at first these episodes had occurred 2 or 3 times a day, they had progressed to $>10$ times a day after 2 weeks. No signs of insomnia or hyponatremia were observed. The patient's temperature was normal and her past medical history was unremarkable. The patient had no vomiting and headache at the onset of her condition. No focal deficit was found on neurological examination, with the exception of memory disturbance. Cranial magnetic resonance (MR)-diffusion weighted imaging and -fluid attenuated inversion recovery imaging revealed a hyperintense signal in the left hippocampus and right basal ganglia, but without contrast enhancement (Figs. 1 and 2). An electroencephalogram (EEG) revealed rhythmic sharp and slow waves and rhythmic $\theta$ build-ups in the left temporal area (Fig. 3). Video-EEG captured eleptiform paradoxical discharge when the patient was asleep at night. Single-photon emission computed tomography (SEPCT) showed regional blood flow perfusion in the left cerebral frontal lobe and right basal ganglia. Laboratory studies revealed a normal serum sodium level, and the glucose, lactate and protein levels in the cerebrospinal fluid (CSF) were normal. No evidence of herpes simplex virus 1 or 2, Borrelia burgdorferi or Treponema pallidum was found in the CSF or the blood. The serum and CSF were positive for antibodies to LGI1 but negative for anti-Yo, -Ri, -Hu, -Ma, -N-methyl-D-aspartate receptor, 


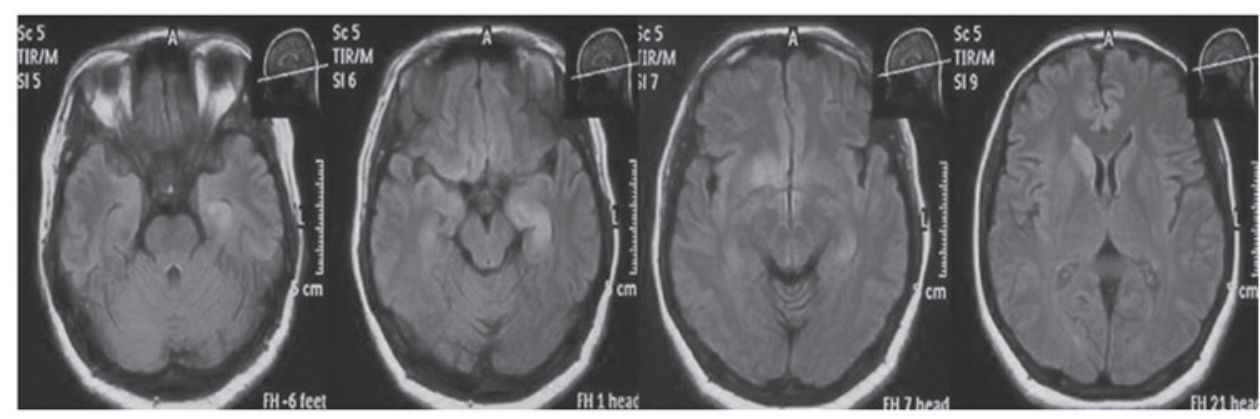

Figure 1. Cranial magnetic resonance-fluid attenuated inversion recovery imaging showed a hyperintense signal in the left hippocampus and right basal ganglia.

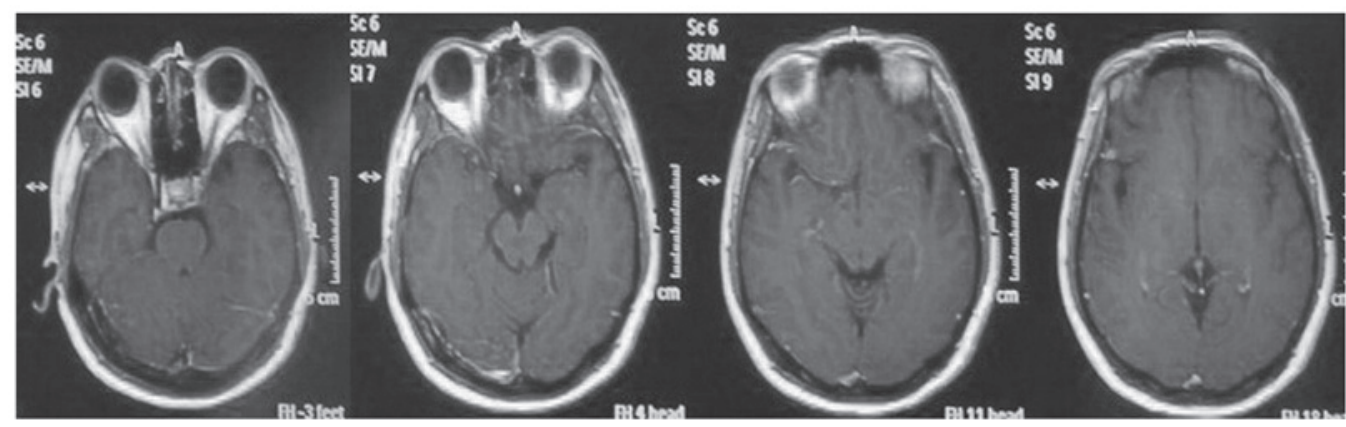

Figure 2. No abnormal signal was observed on the cranial magnetic resonance imaging enhancement scan.

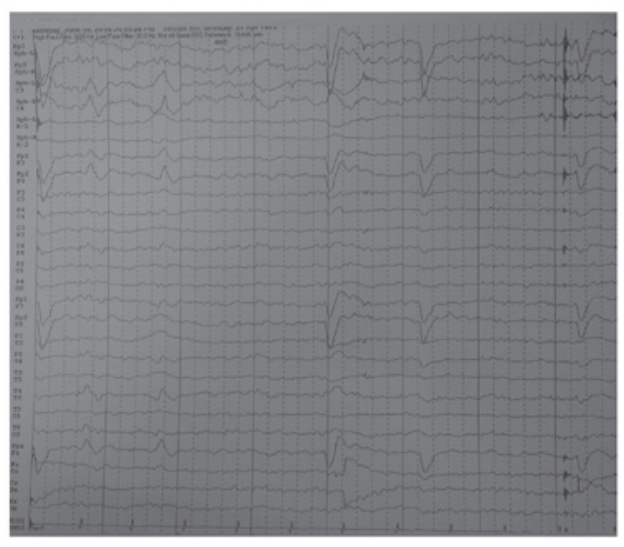

Figure 3. An electroencephalogram revealed rhythmic sharp and slow waves and rhythmic $\theta$ build-ups in the right temporal area.

- $\alpha$-amino-3-hydroxy-5-methyl-4-isoxazolepropionic acid receptor and $-\mathrm{CV} 2$ autoantibodies.

The patient was diagnosed with anti-LGI1 LE based on the characteristic FBDSs, memory loss and positive LGI1 antibodies in the blood and CSF. A treatment regimen of $500 \mathrm{mg} /$ day IV methylprednisolone for 3 days followed by $250 \mathrm{mg}$ /day for 3 days and $125 \mathrm{mg}$ /day for 3 days was initiated. This was followed by IVIg $(0.4 \mathrm{~g} / \mathrm{kg} /$ day $)$ for 5 days and 8 weeks of tapered oral prednisolone. The patient also received oxcarbazepine. The FBDSs of the patient stopped completely 1 day after the initiation of treatment and her memory deficits improved. At 3 months after treatment began, the patient remained free from epileptic seizures and her memory had been partially restored.

\section{Discussion}

LE is a well-recognized syndrome and is associated with several different antibodies, including anti-Hu, anti-Ri, anti-Yo, anti-Ma2, anti-amphiphysin and anti-CV2/collapsin response mediator protein 5 . These antibodies are expressed throughout the nervous system and are also associated with less well-known neurological disorders that affect wider brain systems (5). Numerous patients with LE do not have detectable brain tumors.

Anti-LGI1 LE has been identified as an autoimmune encephalitis. The disorder usually involves the medial temporal area, which causes memory dysfunction and seizures, and has distinctive clinical characteristics, including FBDSs, memory disturbance and a subacute, progressive course (6). According to the literature, hyponatremia is commonly found in patients with anti-LGI1 LE (1); however, this is a non-specific sign (7). The patient in the present case exhibited the characteristic clinical symptoms, but no hyponatremia, insomnia or abnormalities on cranial MR imaging, video-EEG and SPECT. The patient's diagnosis was confirmed by the presence of LGI1 antibodies in the blood and CSF.

The diagnosis of autoimmune LE is difficult and often delayed. While certain cases involve the limbic system exclusively, other systems may also be involved, confusing the diagnostic picture (8). Clinicians in Korea (5) described a case that spontaneously went into remission prior to a definitive diagnosis being made. The symptoms recurred in 2013, when the disorder was identified. French researchers observed a 65-year-old anti-LGI1 LE patient with insomnia in 2012 (9). Only few reports have highlighted the presence of reversible insomnia in autoimmune encephalitis (10), and the 
mechanisms by which LGI1 antibodies may cause insomnia remain unclear (9). German researchers were the first to report the neuropathological characteristics of LGI1 LE and suggested a CD8 ${ }^{+}$T-cell-mediated immune process directed against hippocampal neurons (11).

Early diagnosis of this rare disease is important so that treatment can be instituted as soon as possible. Treatment delays can result in ongoing functional memory problems and other lingering neurological deficits (12). FBDSs may be the earliest manifestation of LE associated with the LGI1 antibody, so recognizing this unique type of seizure at once is important and allows treatment to start quickly, when it is most effective (13).

Although there are no definitive therapeutic guidelines for anti-LGI1 LE (14), first-line treatment usually involves high doses of steroids in combination with other immunosuppressive therapy, such as IVIg, plasma exchange or mycophenolate (15). Failure to respond within 1-2 weeks should prompt second-line therapy, such as three to five plasmapheresis exchanges or the administration of cyclophosphamide or rituximab (16). Response to therapy is best judged by the patient's clinical status. Following antibody titers alone is not useful, as they do not always correlate with clinical progress. The effect of long-term, steroid-sparing agents, such as mycophenolate or azathioprine, on relapse rates is unclear (17).

If clinicians suspect anti-LGI1 LE due to symptoms of FBDSs and memory loss, immunotherapy should begin at once, even before the laboratory results have confirmed the presence of LGI1 antibodies in the CSF and blood, to limit the duration of the illness.

\section{References}

1. Irani SR, Michell AW, Lang B, Pettingill P, Waters P, Johnson MR, Schott JM, Armstrong RJ, S Zagami A, Bleasel A, et al: Faciobrachial dystonic seizures precede Lgil antibody limbic encephalitis. Ann Neurol 69: 892-900, 2011.

2. Asztely F and Kumlien E: The diagnosis and treatment of limbic encephalitis. Acta Neurol Scand 126: 365-375, 2012.
3. Tüzün E and Dalmau J: Limbic encephalitis and variants: Classification, diagnosis and treatment. Neurologist 13: 261-271, 2007.

4. Lancaster E, Huijbers MG, Bar V, Boronat A, Wong A, Martinez-Hernandez E, Wilson C, Jacobs D, Lai M, Walker RW, et al: Investigations of caspr2, an autoantigen of encephalitis and neuromyotonia. Ann Neurol 69: 303-311, 2011.

5. Lee JJ, Lee ST, Jung KH, Chu K and Lee SK: Anti-lGI1 Limbic encephalitis presented with atypical manifestations. Exp Neurobiol 22: 337-340, 2013.

6. Machado S, Pinto AN and Irani SR: What should you know about limbic encephalitis? Arq Neuropsiquiatr 70: 817-822, 2012.

7. Gulati S and Kumar L: 'Chest epilepsy' in a child. Postgrad Med J 68: 369-370, 1992.

8. Irani SR, Bien CG and Lang B: Autoimmune epilepsies. Curr Opin Neurol 24: 146-153, 2011.

9. Peter-Derex L, Devic P, Rogemond V, Rheims S, Mauguière F and Honnorat J: Full recovery of agrypnia associated with anti-Lgi1 antibodies encephalitis under immunomodulatory treatment: A case report with sequential polysomnographic assessment. Sleep Med 13: 554-556, 2012.

10. Montiel P, Sellal F, Clerc C, Richard P and Batailard M: Limbic encephalitis with severe sleep disorder associated with voltage-gated potassium channels (VGKCs) antibodies. Rev Neurol (Paris) 164: 181-184, 2008 (In French).

11. Schultze-Amberger J, Pehl D and Stenzel W: LGI-1-positive limbic encephalitis: A clinicopathological study. J Neurol 259: 2478-2480, 2012

12. Irani SR, Stagg CJ, Schott JM, Rosenthal CR, Schneider SA, Pettingill P, Pettingill R, Waters P, Thomas A, Voets NL, et al: Faciobrachial dystonic seizures: The influence of immunotherapy on seizure control and prevention of cognitive impairment in a broadening phenotype. Brain 136: 3151-3162, 2013.

13. Sen A, Wang J, Laue-Gizzi H, Lee T, Ghougassian D and Somerville ER: Pathognomonic seizures in limbic encephalitis associated with anti-LGI1 antibodies. Lancet 383: 2018, 2014.

14. Thieben MJ, Lennon VA, Boeve BF, Aksamit AJ, Keegan M and Vernino S: Potentially reversible autoimmune limbic encephalitis with neuronal potassium channel antibody. Neurology 62: $1177-1182,2004$.

15. Lancaster E, Martinez-Hernandez E and Dalmau J: Encephalitis and antibodies to synaptic and neuronal cell surface proteins. Neurology 77: 179-189, 2011.

16. Ishiura H, Matsuda S, Higashihara M, Hasegawa M, Hida A, Hanajima R, Yamamoto T, Shimizu J, Dalmau J and Tsuji S: Response of anti-NMDA receptor encephalitis without tumor to immunotherapy including rituximab. Neurology 71: 1921-1923, 2008.

17. Irani SR and Vincent A: NMDA receptor antibody encephalitis. Curr Neurol Neurosci Rep 11: 298-304, 2011. 